\title{
Three-Dimensional (3D) Imaging of Tumor Angiogenesis and its Inhibition: Evaluation of Tumor Vascular-Targeting Agent Efficacy in the DMBA-induced Rat Breast Cancer Model by Confocal Laser Scanning Microscopy (CLSM)
}

\author{
Johbu Itoh ${ }^{1}$, Kazuhiko Yasumura ${ }^{2}$, Kenji Ogawa ${ }^{3}, K_{\text {Kenji Kawai }}^{4}$, Akihiko Serizawa ${ }^{4}$, \\ Yukito Yamamoto ${ }^{5}$ and Yoshiyuki R. Osamura ${ }^{6}$ \\ ${ }^{1}$ Laboratories for Structure and Function Research, ${ }^{4}$ Division of Diagnostic Pathology, ${ }^{6}$ Department of Pathology, Tokai University, \\ School of Medicine, Isehara, Kanagawa 259-1193, Japan, ${ }^{2}$ Department of Surgery, ${ }^{3}$ Department of Diagnostic Radiology, \\ ${ }^{5}$ Pathological Division of Clinical Laboratory, NKK Hospital, Kawasaki, Kanagawa 210-0852, Japan
}

Received February 21, 2003; accepted February 28, 2003

There is considerable interest in tumor angiogenesis and its inhibition, because tumor angiogenesis plays an important role in tumor growth and distant metastasis, and is highly associated with overall survival and relapse free-survival in cancer patients.

This study was performed to determine the mechanisms underlying tumor angiogenesis and its inhibition using the angiogenesis inhibitor TNP 470 and antimitotic drug, docetaxel (Taxotere). Variable 3D image analysis of tumor vasculature after chemotherapy was performed by CLSM using the DMBA-induced rat breast cancer model.

3D images of the vasculature in DMBAinduced rat breast cancer were charac- terized by increased microvessel density and anastomosing, irregularly branched microvessel networks. After administration of TNP-470 or docetaxel, tumor microvessel density was markedly decreased and tumor microvessel networks were markedly destroyed. Furthermore, tumor microvessels less than $30 \mu \mathrm{m}$ in diameter were not found. The remaining tumor microvessels $(30-60 \mu \mathrm{m}$ in diameter) were mainly localized in the periphery of the tumor. These observations suggest that docetaxel might have the ability to inhibit tumor angiogenesis as well as TNP-470. CLSM with the FI method is a valuable technique for estimation of the efficacy of anti-angiogenic chemotherapy.

Key words: tumor angiogenesis, tumor vascular-targeting agent, DMBA rat mammary cancer, FITC-conjugated gelatin injection method, CLSM

\section{Introduction}

There has been considerable interest in tumor angiogenesis and its inhibition, because tumor angiogenesis plays an important rule in tumor growth itself, distant metastasis and is highly associated with overall survival and relapse free survival in cancer patients. It is well known that the solid tumor requires formation of new blood vessels (angiogen-

Correspondence to: Johbu Itoh, Ph.D., Laboratories for Structure and Function Research, School of Medicine, Tokai University, Bohseidai, Isehara, Kanagawa 259-1193, Japan. esis), for its progression [4, 13]. Most of the research efforts in this area have focused on understanding the process of angiogenesis and identifying anti-angiogenic argents. Currently, these are several classes of anti-angiogenic agents in phase II/III clinical trials for cancer, including inhibitions of matrix-metallo-proteinase, agents that inhibit vascular endothelial growth factor or its receptor, and inhibitors of endothelial growth such as TNP-470 [27, 45]. Other agents that have shown promise in pre-clinical evaluation include the peptide inhibition of endothelial cell proliferation, angiostatin and endostatin. [8, 37].

In contrast to the anti-angiogenesis approach, anti- 
vascular approaches aim to cause a rapid and extensive shut-down of the established tumor vasculature, leading to secondary tumor cell death. Development of antibody to specific epitopes on tumor vasculature [20] and vasculartargeted gene therapy [7] are two approaches that are receiving considerable attention.

However, drug-and cytokine-based approaches to vascular targeting are also possible. Two main classes of drugs can include vascular damage and subsequent hemorrhagic necrosis in tumors, mainly the tubulin-binding agents $[6,7]$ and drugs related to flavone acid [19].

Therefore it is thought that inhibition of angiogenesis might be clinically useful for therapy of solid tumors $[12,15$, $16,35]$. Folkman reported the concept of treating tumors by inhibiting their ability to resist new blood microvessels [14]. On the other hand, there has been considerable interest in angiogenic molecules. For example, vascular endothelial growth factor (VEGF) stimulates endothelial cells directly and results in a potent inducer of tumor angiogenesis $[3,5,25,36,39,40,42]$. Recently studies revealed that cancer cells themselves secrete various angiogenic molecules including VEGF and anti-cancer drugs efficacy $[11,18,28$, 46]. Among them, the fumagillin analogue TNP-470 is a selective inhibitor of endothelial cell proliferation and migration and is under investigation in clinical trials as an anticancer agent $[2,17,48,50]$.

TNP-470 inhibits primary tumor growth and demonstrates $70 \%$ inhibition of angiogenesis in the corneal micropocket assay [21]. Docetaxel (taxotere) is antimicrotubule agents effective in the treatment of common cancers in humans $[1,9,30,32,38,43,49]$. They are mitotic spindle poisons and promote accelerated assembly of excessively stable microtubules, thus affecting microtubule-dependent cellular functions like the control of mitosis and intracellular transport. On the other hand, antimitotic drugs (the majority of these agents induce mitotic arrest by interacting with tubulin assembly) have been so successful in the treatment of cancer that no one would dispute that tubulin is a valid target. However, the evaluations of tumor angiogenesis inhibition by antimitotic chemotherapy have not been studied extensively.

Previously, we had reported a new technique of 3D vasculature analysis by confocal laser scanning microscopy (CLSM) using 7,12-dimethylbez (a) anthracene (DMBA)induced rat mammary cancer model [26, 44, 47], named "fluorescence injection (FI) method" [22-25]. This FI technique with CLSM clearly demonstrates $3 \mathrm{D}$ vasculature and dynamic functional images of tumor angiogenesis.

This study was aimed at revealing the underlying mechanisms of tumor angiogenesis and their inhibition using angiogenic inhibitor TNP 470 and antimitotic drug, docetaxel (Taxotere). Variable 3D image analysis of tumor vasculatures and angiogenic, proliferating status of the tumor after chemotherapy were evaluated by CLSM using FI method with DMBA-induced rat mammary cancer model.

\section{Materials and Methods}

Eighteen female normal adult Sprague-Dawley (SD) rats weighing 150-190 g were fed every 4 weeks with $20 \mathrm{mg}$ 7,12-dimethylbez (a) anthracene (DMBA) dissolved in corn oil through a gastric tube. After 8-12 weeks, DMBAinduced rats, whose mammary tumors measured $2.0 \mathrm{~cm}$ at their widest diameter, were selected and used for the experiment. Tumor size and body weight were measured before treatment and daily thereafter. Six DMBA-induced rats were used as controls. Six DMBA-induced rats given taxotere were also injected intravenously (I.V.) every two days for 3 times with $5.0 \mathrm{mg} / \mathrm{kg}$. Another 6 DMBA-induced rats; TNP470 was dissolved in $5 \%$ Arabic gum and $40 \mathrm{mg} / \mathrm{kg}$ and was subcutaneously administered every two days for 3 times. For the control study, untreated, age-matched female SD rats were used.

\section{FITC-conjugated gelatin injection method}

Fluorescein-5-isothiocyanate (FITC) was obtained from Dojindo (Kumamoto, Japan) and gelatin from Sigma Chemical (St Louis, MO, USA). FITC was conjugated to gelatin by brief reaction at $37^{\circ} \mathrm{C}$ and $\mathrm{pH} 11$. Typically, $10-$ $50 \mathrm{mg}$ of FITC was dissolved in 1-ml dimethylsulfoxide (DMSO; Sigma), pH 11. A 20\% gelatin solution made with distilled water, $\mathrm{pH} 11$ and DMSO containing FITC was mixed for conjugation at $37^{\circ} \mathrm{C}$ overnight. Then FITCconjugated gelatin was dialyzed in $0.01-\mathrm{M}$ phosphate buffered saline (PBS), pH 7.4 at $37^{\circ} \mathrm{C}$ in the dark for 1 week. Aliquots of the dialyzed FITC-conjugated gelatin in a total volume of $200-\mathrm{ml}$ were injected into the rat.

\section{Tissues preparations}

The abdominal and chest walls of the animals were opened under diethyl ether inhalation anesthesia. To demonstrate microvessels, two hundred $\mathrm{ml}$ of dialyzed FITC (30$\mathrm{mg} / \mathrm{ml}$ )-conjugated gelatin solution was injected into the left ventricle of the heart, cutting off the atrium dextrum. After complete perfusion within approximately 3-5 $\mathrm{min}$, the animals were fixed in cold 4\% paraformaldehyde (PFA, Wako Pure Chemical, Osaka, Japan) added to $15 \%$ picric acid (Wako) solution $\left(0^{\circ} \mathrm{C}\right)$ over approximately $25-30 \mathrm{~min}$. Then, wet mammary glands were resected and immediately fixed in graded cold PFA $(0.5-8 \%)$ containing $15 \%$ picric acid overnight $\left(4^{\circ} \mathrm{C}\right)$.

For the simultaneous visualization of microvessel networks and immunohistochemical localization of keratin, VEGF and CD-34, the mammary tissues were cut into 2$3 \mathrm{~mm}$ thick blocks, placed on $50 \mathrm{~mm} \times 70-\mathrm{mm}$ cover glasses (Matsunami glass INO. Osaka, Japan), and mounted in $0.05 \mathrm{M}$ Tris- $\mathrm{HCl}$ buffer, 1,4-diazabicyclo [2,2,2] octane (DABCO; Wako). The mammary tissue specimens were used for frozen sections. These frozen sections were obtained from tissues that had been fixed overnight in 4\% PFA dissolved in 0.01 M PBS, $\mathrm{pH} 7.4$, immersed in graded concentrations of sucrose in PBS $(10 \%$ for $1 \mathrm{hr}, 15 \%$ for $2 \mathrm{hr}$ and $20 \%$ for $4 \mathrm{hr}$ ), embedded in OCT compound (Tissue-Tek; 

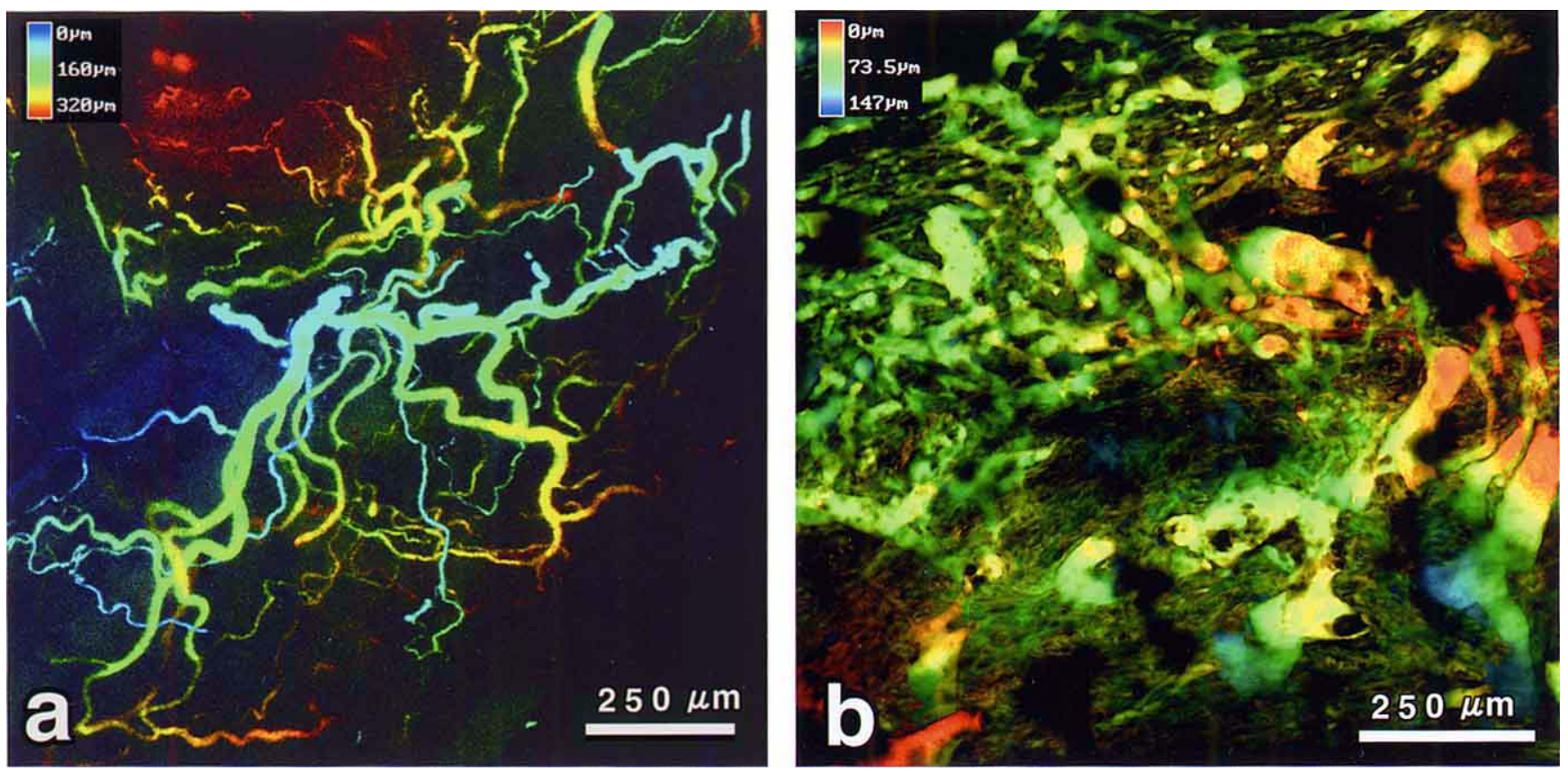

Fig. 1. 3D color depth focus imaging of microvessel networks (MV) by CLSM in a normal rat mammary gland (a) and DMBA-induced rat mammary cancer $(\mathbf{b})$. a: MV showed a regular linear branching pattern. b: The MV density was markedly increased and irregularly branched MV were prominent. The colored gradation scale indicates the focal depth. Bars $=250 \mu \mathrm{m}$.
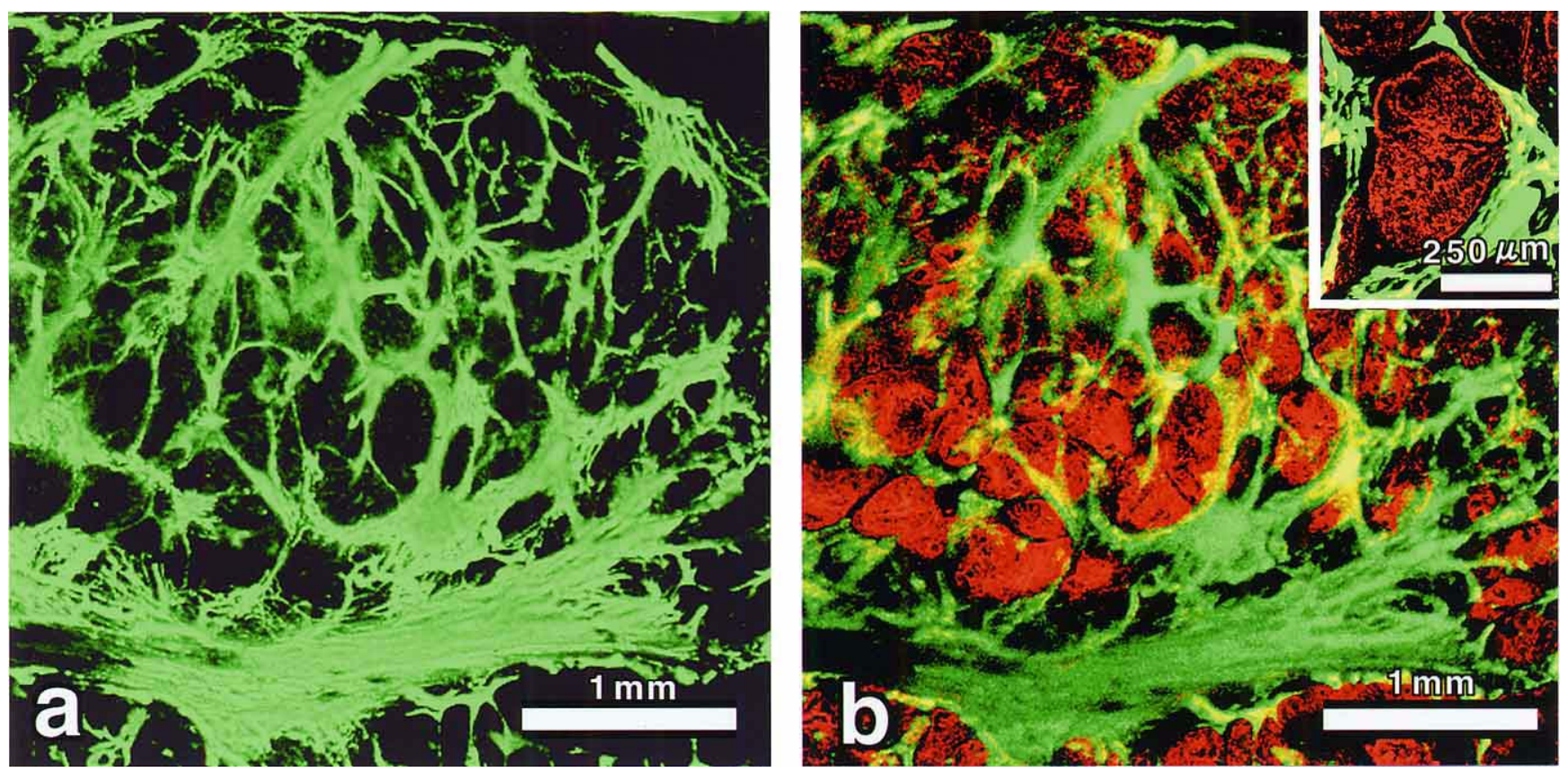

Fig. 2. 3D projection images of MV by CLSM in DMBA-induced rat mammary cancer. a: 3D single projection imaging of MV. The MV density was markedly increased and irregularly branched MV were prominent (Green color; MV). b: 3D double projection imaging of MVN and tumor nests. The relationship between MV and tumor nests was clearly demonstrated by double colored 3D imaging, (Yellow-green color; MV, Red color; tumor nest). Bar=1 mm. Inset: Bar=250 $\mu \mathrm{m}$. 

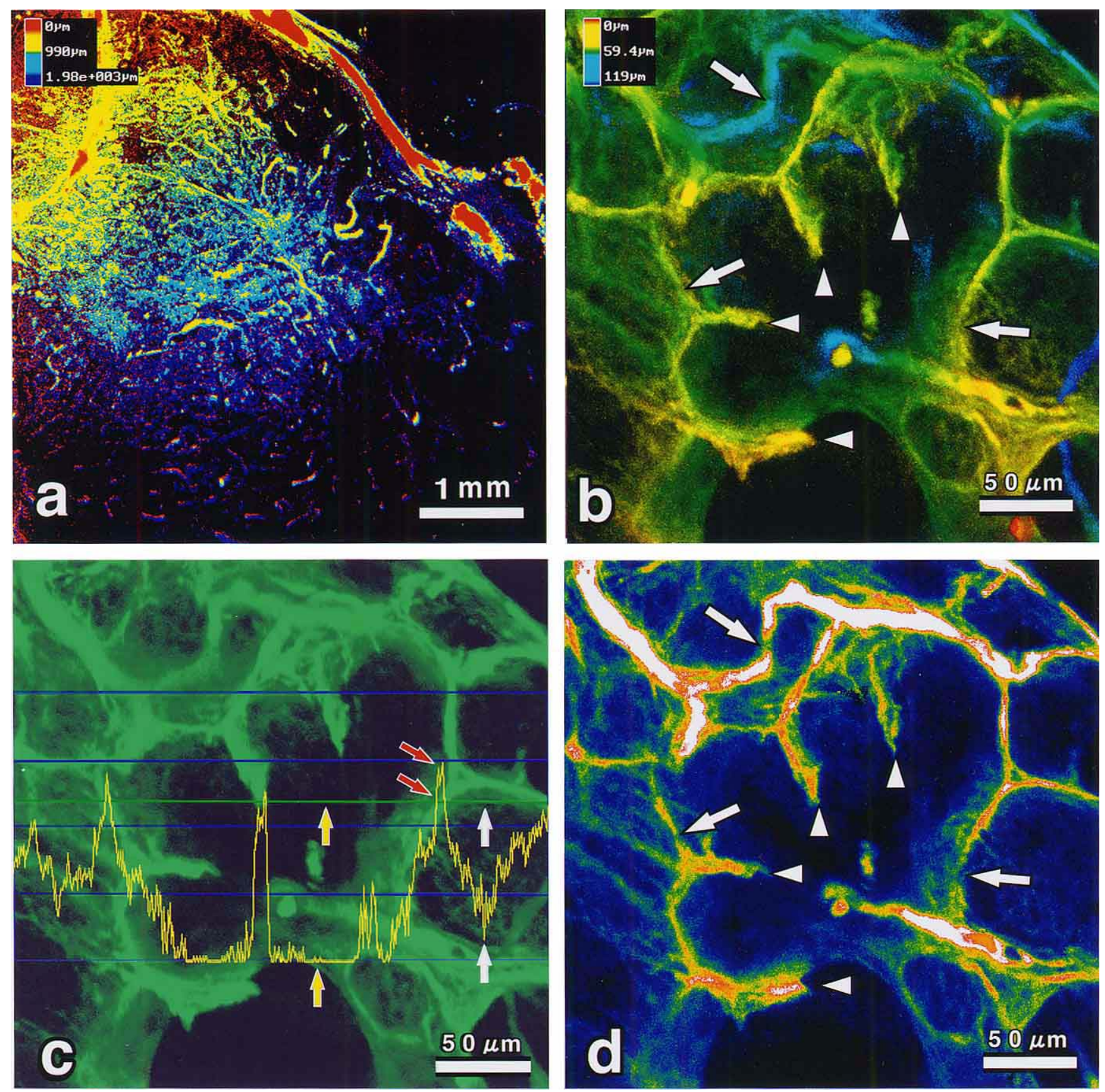

Fig. 3. 3D reconstructed images of DMBA-induced rat mammary cancer. a: Low power $3 \mathrm{D}$ colored depth focus image. The colored gradation scale indicates the focal depth. Bar=1 mm. b: High power 3D colored depth focus image. Some microvessels showed abnormalities such as an angular course with focal narrowing (arrows) and stoppage ends pattern (arrowheads). Bar $=50 \mu \mathrm{m}$. c: $3 \mathrm{D}$ projection images of MV and signal intensity profile of MV. FITC gelatin was detected in the extravascular space (leaked FITC gelatin; white arrows) as well as in the intravascular space (hyperpermeable microvessels; red arrows). Extravasation of FITC gelatin was not detected (no leaked FITC gelatin in tumor nest; yellow arrows). Color scale imaging of signal intensity of MV showed morphological and physiological tumor microvessel characteristics. The green line indicates the scanning position of the single intensity. Bar $=50 \mu \mathrm{m}$. d: Colored scale imaging of signal intensity of MV. Some microvessels showed abnormalities such as an angular course with focal narrowing (arrows) and stoppage ends pattern (arrowheads). Various degrees of extravasation of FITC gelatin from microvessels were demonstrated by color scale imaging. Bar $=50 \mu \mathrm{m}$. 
Miles Laboratories, Elkhart, IN, USA) and stored at $-80^{\circ} \mathrm{C}$ until use. Thirty $\mu \mathrm{m}$-thick frozen sections were mounted on 3-amino-propylmetho-xysilane-coated slides.

\section{Immunohistochemistry}

An immunohistochemical analysis of tumor cells was performed by indirect immunofluorescent and immunoperoxidase labeled antibody methods. The procedure was as described by Nakane [34].

The first antibody employed was rabbit polyclonal antiVEGF antibody (1:200, Chemicon International, Temecula, CA, USA), mouse monoclonal anti-CD-34 antibody (1:200,
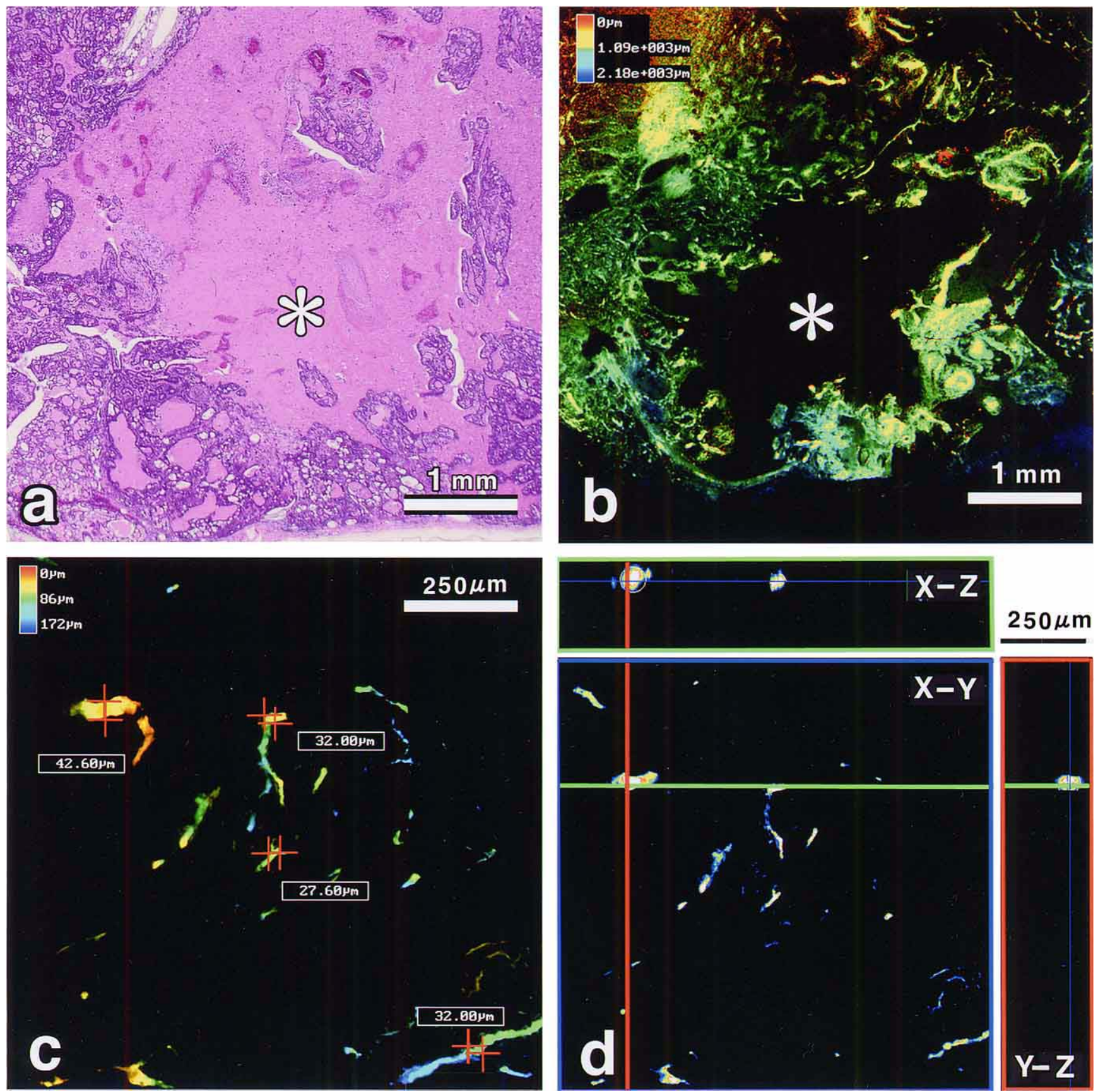

$250 \mu \mathrm{m}$
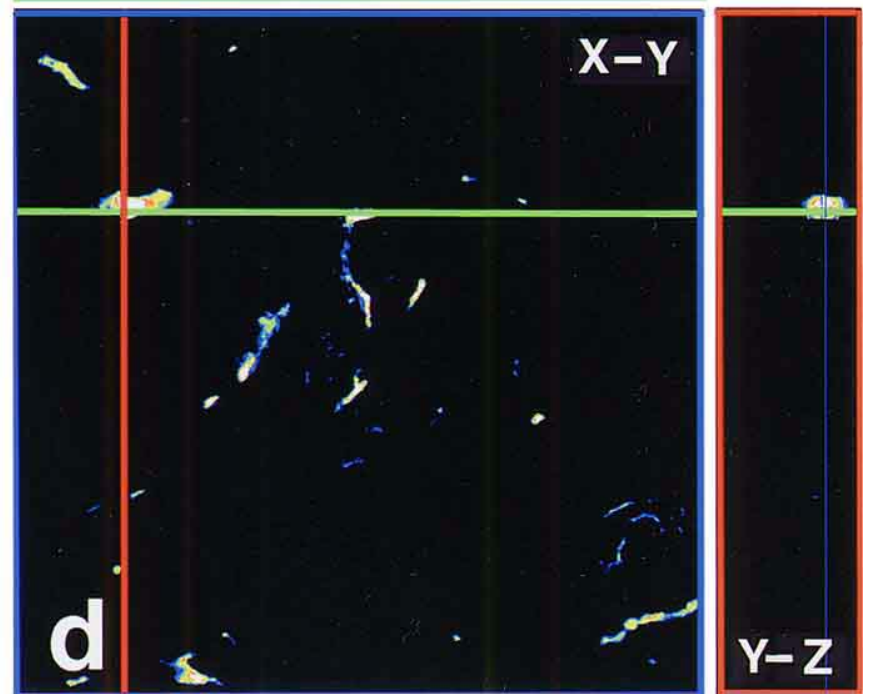

Fig. 4. 3D reconstructed images of tumor microvessels in DMBA-induced rat mammary cancer with docetaxel (taxotere) administration by CLSM. a: Light microscopic histological observation (HE staining). b, c: 3D colored depth focus images of tumor microvessels. d: 3D colored intensity orthogonal section image of tumor microvessels. X-Y cross-section plane image (blue line square), X-Z cross-section plane image (green line square), Y-Z cross-section plane image (red line square). Tumor microvessel density was markedly decreased and tumor microvessel networks were markedly destroyed. Furthermore, tumor microvessels less than $30 \mu \mathrm{m}$ in diameter were not found. $(*)$; necrotic lesion. Bars $=1$ $\mathrm{mm}(\mathbf{a}, \mathbf{b}), 250 \mu \mathrm{m}(\mathbf{c}, \mathbf{d})$. 

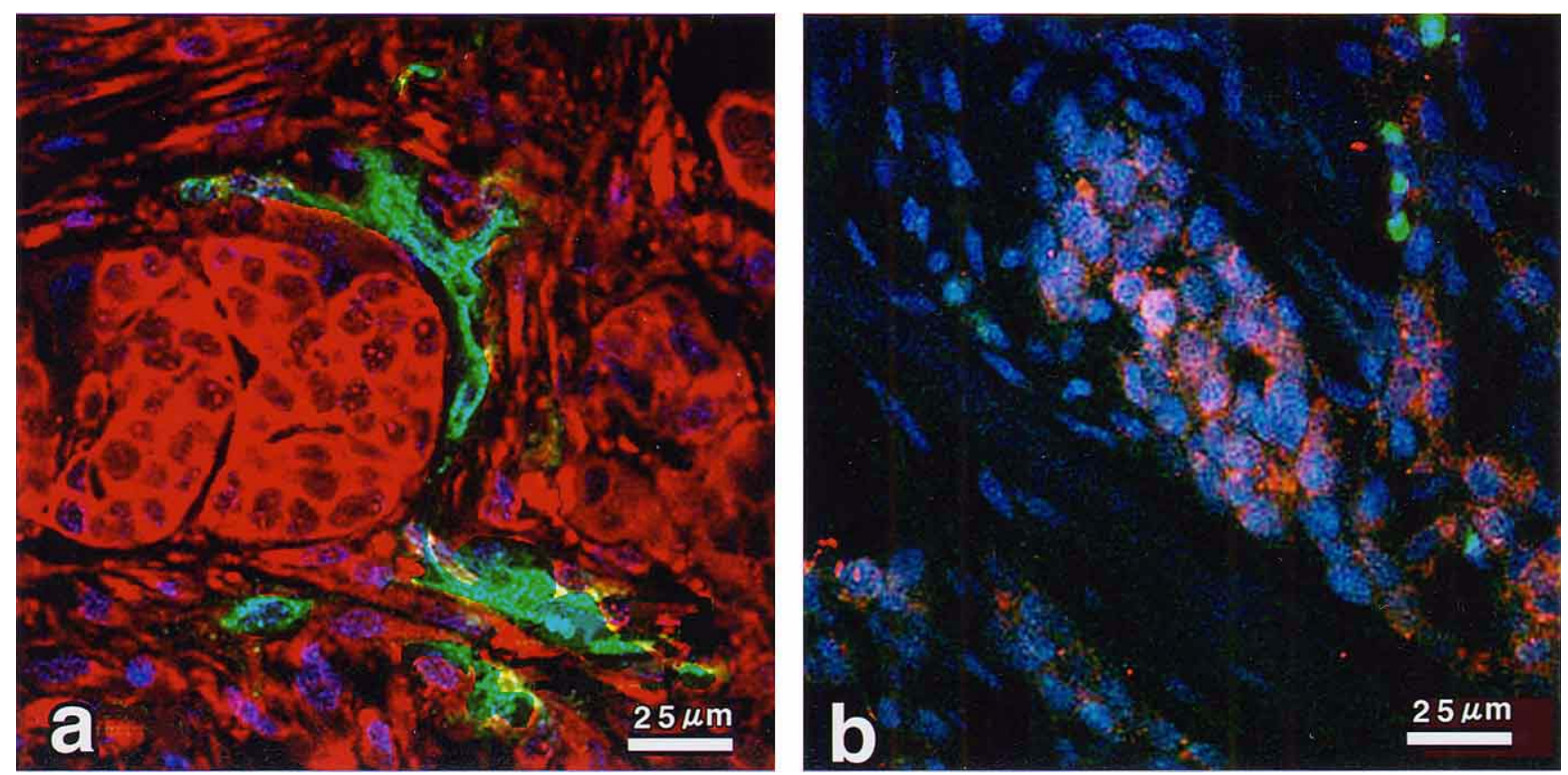

Fig. 5. Comparison of 3D reconstructed images of docetaxel (taxotere) treated and untreated DMBA-induced rat mammary cancer. a: Docetaxel (Taxotere) pre-treatment. Three-dimensional projection image of tumor cells and MV in docetaxel (taxotere) pretreatment animals. Many tumor cells were closely related with microvessels. Red; VEGF (TRITC), Yellow; CD-34 (endothelial cells; FITC), Blue; Nuclei (DAPI). b: Docetaxel (Taxotere) post-treatment. Three-dimensional projection image of tumor cells and MV in Docetaxel (Taxotere) post-treatment. Red; VEGF (TRITC), Yellow; CD-34 (endothelial cells; FITC), Blue; Nuclei (DAPI). MV had markedly increased in size and number and is inhibiting which indicates an anti-cancer drug effect. Bars $=25 \mu \mathrm{m}$.

Nobocastra Laboratories, Newcastle upon Tyne, UK) and rabbit polyclonal anti-keratin wide spectrum (DAKO, Carpinteria, CA, USA). The second antibody employed was (1) tetramethylrhodamine isothiocyanate (TRITC)-conjugate swine anti-rabbit immunoglobulins (1:10, DAKO), or (2) horseradish peroxidase (HRP)-conjugate sheep antimouse (1:50, Amersham, UK). The peroxidase coloring reaction was performed with 3-3' diaminobenzidine (DAB; Dojindo). Then the nuclei were counterstained with $5 \%$ methyl green $(\mathrm{MG})$ buffered by $0.1-\mathrm{mol} / \mathrm{L}$ Veronal acetate at pH 4.0 (Chroma, Netherlands) and/or 4',6-diamidino-2phenylindole (DAPI) $(1 \mathrm{mg} / \mathrm{ml}) 1: 1000$ dilution for $60 \mathrm{~min}$ at room temperature.

\section{Confocal laser scanning microscopic (CLSM) system}

Confocal laser scanning microscopy (LSM-410, Carl Zeiss, Jena, Germany) was employed on thick sections (1-2 $\mathrm{mm}$ ) to elucidate the relation between tumor cells and the microvessel network by colored depth focus, colored intensity profile, projection and $3 \mathrm{D}$ cutting at reconstruction modes. The system included a 351/364 nm Argon UV laser (for DAPI) and a $488 \mathrm{~nm}$ Argon laser (for FITC) and/or a $543 \mathrm{~nm}$ Helium Neon laser (for TRITC and DAB). Optical fluorescence signals of microvessel network patterns labeled by FITC were observed using a dichroic beam splitter (NT 80/20/543, Carl Zeiss) and emission filter (BP510-525, Carl Zeiss). Optical fluorescence signals of keratin, VEGF, CD-
34 labeled by TRITC were observed using a dichroic beam splitter (NT 80/20/543, Carl Zeiss) and emission filter (LP570, Carl Zeiss). Optical reflectance signals of CD 34 labeled by HRP were observed using a dichroic beam splitter (NT 80/20/543, Carl Zeiss) and no emission filter. Optical fluorescence signals of nuclei labeled by DAPI were observed using a dichroic beam splitter (NT 80/20/543, Carl Zeiss) and emission filter (LP397, Carl Zeiss). Double or triple-labeled volumetric data sets of these images taken with confocal laser scanning microscopy can be digitized and made susceptible to image analysis manipulation and computer-assisted 3D reconstructions using LSM software version 3.98 (Carl Zeiss, Jena, Germany).

The optical tomographic imaging (Z-images) for 3D reconstruction of tumor cells and their microvessels was done with a C-apochromat $(\times 63$, water, N.A. 1.25, Carl Zeiss $)$ and a plan-neofluar $(\times 10$, N.A. $0.30 ; \times 20$, N.A. 0.50, Carl Zeiss $)$ objective lens, and a stepping motor unit for axial scanning at $0.5-20$ controlled $Z$ directional movement for the optical sectioning of entire specimens $\mu \mathrm{m}$ focus steps. The image resolution was $512 \times 512$ and/or $1024 \times 1024$ pixels ( 8 bits, 256 gray levels).

Following computer-assisted 3D imaging of tumor cells and their microvessels using the LSM systems, the images were stored on hard disk memory and/or magnetic optical disk EDM-230C (SONY, Japan) and printed with a Pictrostat digital 400 (Fuji film, Japan). 

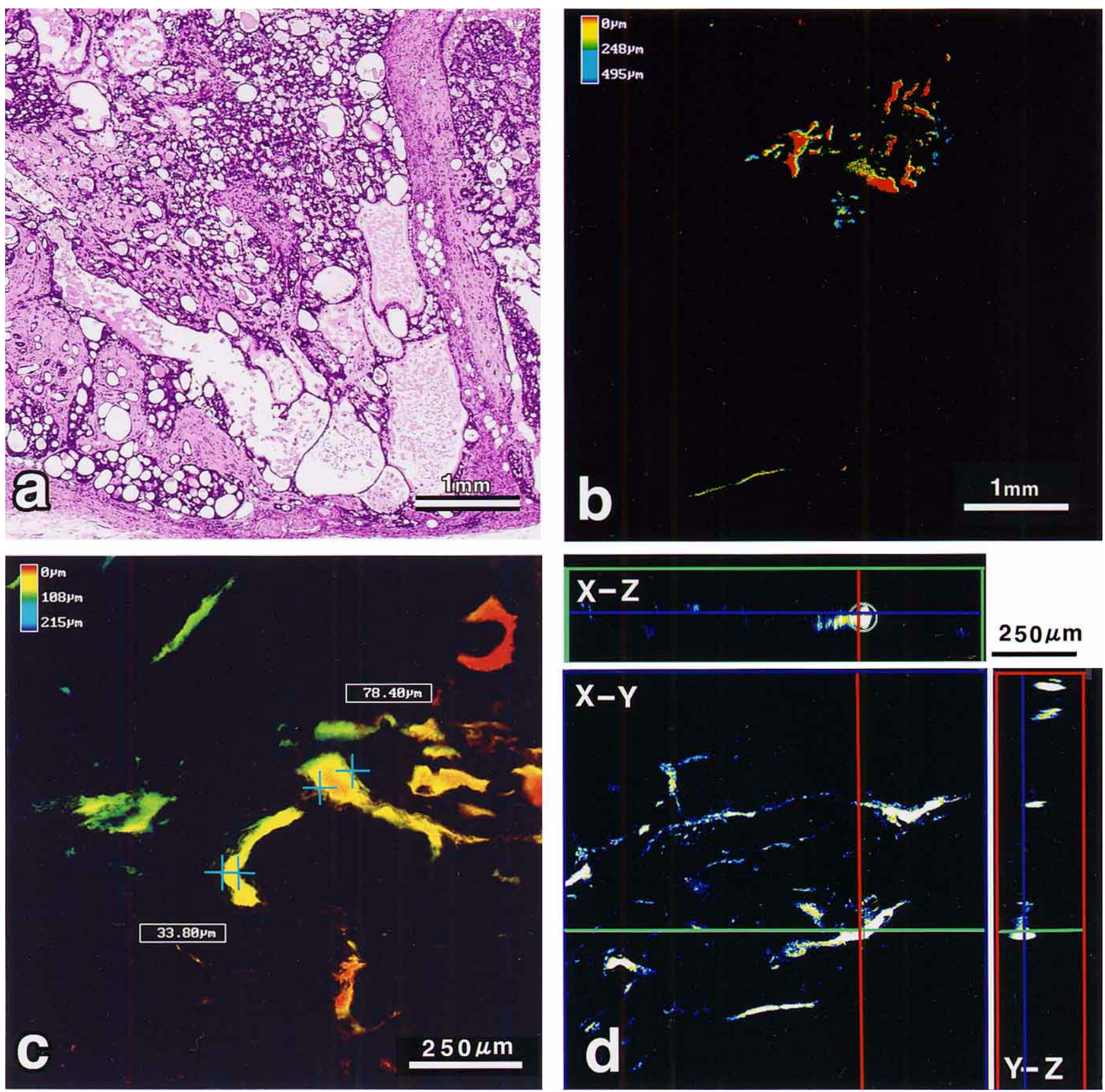

$250 \mu \mathrm{m}$

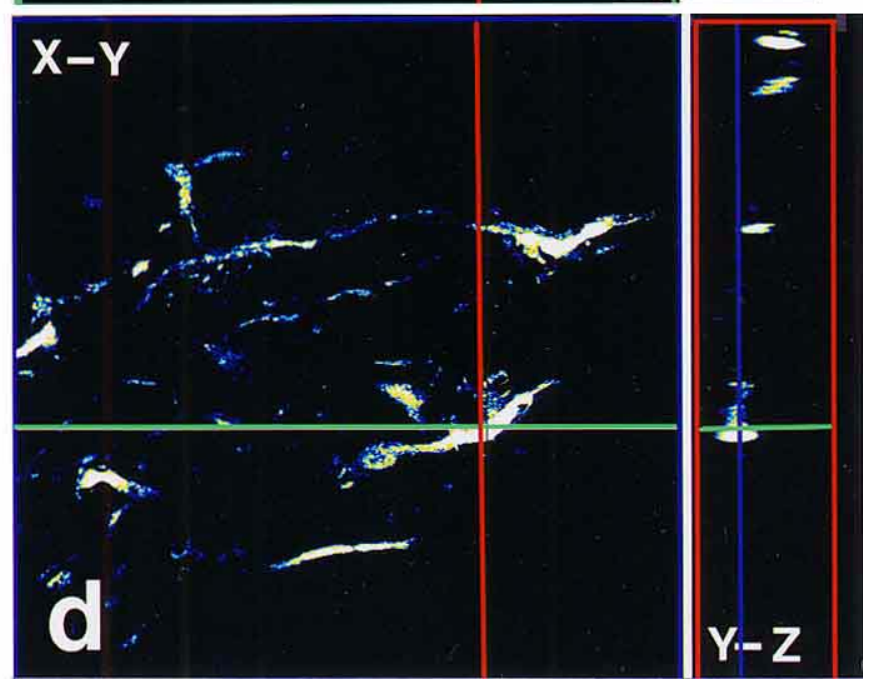

Fig. 6. $3 \mathrm{D}$ reconstructed images of tumor microvessels in DMBA-induced rat mammary cancer with TNP-470 administration by CLSM. a: Light microscopic histological observation (HE staining). b, c: 3D colored depth focus images of tumor microvessels. d: 3D colored intensity orthogonal section image of tumor microvessels. X-Y plane image (blue line square), X-Z plane image (green line square), Y-Z plane image (red line square). Tumor microvessel density was markedly decreased and the tumor microvessel network was markedly destroyed. Furthermore, tumor microvessels less than $30 \mu \mathrm{m}$ in diameter were not found. Bars $=1 \mathrm{~mm}(\mathbf{a}, \mathbf{b}), 250 \mu \mathrm{m}(\mathbf{c}, \mathbf{d})$.

\section{Results}

\section{D microvessels network image by CLSM}

In normal control mammary glands, microvessels network were regular and smooth in shape and showed a linear branching pattern (Fig. 1a). On the other hand, in the DMBA-induced mammary cancer, an additional vascular layer occurred in the boundary between the tumor and the host tissue and vascular density was markedly increased and microvessel network images were distorted, more fine in size and irregular in shape (Figs. 1b, 2a, 3a). 3D images of tumor vasculature showed well-developed networks of microvessels in the DMBA mammary cancer (Figs. 2a, 3a). Focal segmentation and tortuous branching patterns of microvessels were also seen (Figs. 2a, 2b, 3a-3d). Some microvessels showed such abnormalities as an angular 
course with focal narrowing (Fig. 3b, d arrows) and stoppage ends pattern (Fig. 3b-d arrowheads). Direct offshoots occurred with blind ends and multiple branching. Furthermore, FITC signals were frequently observed in the extra vascular space, which were not identified in the mammary glands (Figs. 2b, 3b-3d). However, the signal intensity of the extra vascular space was weaker than that of the intravascular space (Fig. 3c, d)

In DMBA-induced and taxotere and TNP-470 administered rats, tumor microvessel density was markedly decreased and tumor microvessel networks were markedly destroyed (Figs. 4b-4d, 6b-6d). Furthermore, tumor microvessels less than $30 \mu \mathrm{m}$ in diameter were not found (Figs. 4c, 4d, 6c, 6d). The remaining tumor vessels (30-60 $\mu \mathrm{m}$ in diameter) were mainly localized in the periphery lesion of the tumor (Figs. 4b, 6b).

\section{Simultaneous observation of microvessel network and tumor cells}

In DMBA-induced rat, tumor cells immunohistochemically stained with keratin antibody were examined with microvessels network by the CLSM. Two different laser rays selectively detected double labeling signals on the same specimens. The tumor nests were surrounded by distorted and irregularly branched microvessels. Simultaneous study revealed that the tumor nests were closely related with these irregular branching anastomosing microvessels network in 3D (Fig. 2b inset).

\section{Docetaxel (taxotere) administration effect of tumor microvessels in DMBA-induced rat mammary cancer}

Before Docetaxel (Taxotere) administration, the remaining tumor vessels were mainly localized in the periphery lesion of the tumor and VEGF expression was strong (Fig. 5a). After Docetaxel (Taxotere) administration, the remaining tumor vessels were markedly decreased and VEGF expression was weak (Fig. 5b).

\section{Discussion}

Anti-tumor agents of TNP-470 effects have already been reported by Kusaka [31], Moulton [33], and Toi [44]. Anti-mitotic agents of docetaxel (taxotere) effects have been reported by Dykes [10], Klauber [29], and Schimming [41]. These report were also described in two dimensionally.

Our previous study, demonstrated the functional and spatial relationship microvessel network pattern and tumor cells in DMBA-induced rat mammary cancer [22]. This study was aimed reveal the underlying mechanisms of tumor angiogenesis and its inhibition using angiogenic inhibitor TNP 470 and antimitotic drug, docetaxel (Taxotere) in threedimensionally. TNP-470 and docetaxel (Taxotere) administration have a significant effect on the functional and spatial relationship microvessel network pattern and tumor cells in DMBA-induced rat mammary cancer. In DMBA-induced rat mammary cancer, morphologically, vasculature on thick sections was clearly detected by CLSM at the maximum focus depth of $2000 \mu \mathrm{m}$. 3D images of tumor microvessels showed markedly increased high vascular density, anastomosing, and irregular branching microvessels.

Physiological analysis of tumor microvessels network by CLSM is very useful for tumor microvessel characterization. Hyperpermeability is another characteristic property of tumor microvessels. We identified various degrees of fluorescent signals in the extravascular spaces of DMBA induced rat mammary cancer tissue by CLSM. This evidence suggested the possibility of extravasation of FITC gelatin solution from tumor microvessel network to extravascular space. However, this extravasation of FITC gelatin was not detected in normal rat mammary glands. Therefore, macromolecule leakage, extravasation of FITC gelatin seemed to be characteristics of tumor angiogenesis.

\section{In DMBA-induced and TNP-470, Taxotere administered rat}

After administration of TNP-470, tumor microvessel density was markedly decreased and tumor microvessel networks were markedly destroyed. As for the effects of antimitotic chemotherapy by docetaxel, the characteristic features of the tumor cells were aggregation of microtubules in cytoplasm, suppression of VEGF expression, destruction of tumor microvessels and apoptotic bodies.

Furthermore, tumor microvessels less than $30 \mu \mathrm{m}$ in diameter were not found. The remaining tumor microvessels (30-60 $\mu \mathrm{m}$ in diameter) were mainly localized in the periphery area of the tumor. This evidence suggests that docetaxel might have an ability to inhibit tumor angiogenesis as well as TNP-470. The underlying mechanisms of angiogenic inhibition by docetaxel remain unclear, however, CLSM with fluorescent injection method is a useful technique for the evaluation of tumor angiogenesis and its inhibition and will be a state of the art technique for the estimation of antiangiogenic chemotherapy.

The application of this technique would be state of the art to study 3D imaging of tumor angiogenesis as well as clinical application of anti-cancer drug evaluation.

We are currently performing these drugs administrations of time interval analysis to elucidate the early effects in DMBA-induced mammary cancer model.

\section{References}

1. Ascherman, J. A., Knowles, S. L. and Attkiss, K. (2000) Docetaxel (taxotere) extravasation: a report of five cases with treatment recommendations. Ann. Plast. Surg. 45; 438-441.

2. Beecken, W. D., Fernandez, A., Panigrahy, D., Achilles, E. G., Kisker, O., Flynn, E., Joussen, A. M., Folkman, J. and Shing, Y. (2000) Efficacy of antiangiogenic therapy with TNP-470 in superficial and invasive bladder cancer models in mice. Urology $56 ; 521-526$.

3. Berse, B., Brown, L. F., Van de Water, L., Dvorak, H. F. and Senger, D. R. (1992) Vascular permeability factor (vascular endothelial growth factor) gene is expressed differentially in normal tissues, macrophages, and tumors. Mol. Biol. Cell 3; 211220 .

4. Bicknell, R. and Harris, A. L. (1991) Novel growth regulatory factors and tumor angiogenesis. Eur. J. Cancer 27; 781-785. 
5. Brown, L. F., Berse, B., Jackman, R. W., Tognazzi, K., Manseau, E. J., Dvorak, H. F. and Senger, D. R. (1993) Increased expression of vascular permeability factor (vascular endothelial growth factor) and its receptors in kidney and bladder carcinomas. Am. J. Pathol. 143; 1255-1262.

6. Chaplin, D. J., Pettit, G. R., Parkins, C. S. and Hill, S. A. (1996) Antivascular approaches to solid tumour therapy: evaluation of tubulin binding agents. Br. J. Cancer Suppl. 27; S86-88.

7. Chaplin, D. J. and Dougherty, G. J. (1999) Tumour vasculature as a target for cancer therapy. Br. J. Cancer 80 (Suppl 1); 57-64.

8. Cirri, L., Donnini, S., Morbidelli, L., Chiarugi, P., Ziche, M. and Ledda, F. (1999) Endostatin: a promising drug for antiangiogenic therapy. Int. J. Biol. Markers 14; 263-267.

9. Couteau, C., Risse, M. L., Ducreux, M., Lefresne-Soulas, F., Riva, A., Lebecq, A., Ruffie, P., Rougier, P., Lokiec, F., Bruno, R. and Armand, J. P. (2000) Phase I and pharmacokinetic study of docetaxel and irinotecan in patients with advanced solid tumors. J. Clin. Oncol. 18; 3545-3552.

10. Dykes, D. J., Bissery, M. C., Narrison, S. D. Jr and Waud, W. R. (1995) Response of human tumor xenografts in athymic nude mice to docetaxel (RP 56976, Taxotere). Invest. New Drugs 13; $1-11$.

11. Ergun, S., Kilik, N., Ziegeler, G., Hansen, A., Nollau, P., Gotze, J., Wurmbach, J. H., Horst, A., Weil, J., Fernando, M. and Wagener, C. (2000) CEA-related cell adhesion molecule 1: a potent angiogenic factor and a major effector of vascular endothelial growth factor. Mol. Cell 5; 311-320.

12. Folkman, J. and Ingber, D. E. (1987) Angiostatic steroids: method of discovery and mechanism of action. Ann. Surg. 206; 374-383.

13. Folkman, J. (1990) What is the evidence that tumor are angiogenesis dependent? J. Natl. Cancer Inst. 82; 4-6.

14. Folkman, J. (1995) The influence of angiogenesis research on management of patients with breast cancer. Breast Cancer Res. Treat. 36; 109-118.

15. Gagliardi, A., Hadd, H. and Collins, D. C. (1992) Inhibition of angiogenesis by suramin. Cancer Res. 52; 5073-5075.

16. Gasparini, G. (1999) The rationale and future potential of angiogenesis inhibitors in neoplasia. Drugs 58; 17-38.

17. Gervaz, P., Scholl, B., Padrun, V. and Gillet, M. (2000) Growth inhibition of liver metastases by the anti-angiogenic drug TNP470. Liver 20; 108-113.

18. Hagedorn, M. and Bikfalvi, A. (2000) Target molecules for anti-angiogenic therapy: from basic research to clinical trials. Crit. Rev. Oncol. Hematol. 34; 89-110.

19. Hill, S. A., Sampson, L. E. and Chaplin, D. J. (1995) Anti-vascular approaches to solid tumour therapy: evaluation of vinblastine and flavone acetic acid. Int. J. Cancer 63; 119-123.

20. Huang, X., Molema, G., King, S., Watkins, L., Edgington, T. S. and Thorpe, P. E. (1997) Tumor infarction in mice by antibodydirected targeting of tissue factor to tumor vasculature. Science 275; 547-550.

21. Ingber, D., Fujita, T., Kishimoto, S., Sudo, K., Kanamaru, T., Brem, H. and Folkman, J. (1990) Synthetic analogues of fumagillin that inhibit angiogenesis and suppress tumour growth. Nature $348 ; 555-557$.

22. Itoh, J., Yasumura, K., Takeshita, T., Ishikawa, H., Kobayashi, H., Ogawa, K., Kawai, K., Serizawa, A. and Osamura, R. Y. (2000) Three-dimensional imaging of tumor angiogenesis. Anal. Quant. Cytol. Histol. 22; 85-90.

23. Itoh, J., Kawai, K., Serizawa, A., Yasumura, K., Ogawa, K. and Osamura, R. Y. (2000) A new approach to three-dimensional reconstructed imaging of hormone-secreting cells and their microvessel environments in rat pituitary glands by confocal laser scanning microscopy. J. Histochem. Cytochem. 48; 569-578.

24. Itoh, J., Matsuno, A., Yamamoto, Y., Kawai, K., Serizawa, A.,
Watanabe, K., Itoh, Y. and Osamura, R. Y. (2001) Confocal laser scanning microscopic imaging of subcellular organelles, mRNA, protein products, and the microvessel environment. Acta Histochem. Cytochem. 34; 285-297.

25. Itoh, J., Serizawa, A., Kawai, K., Ishii, Y., Teramoto, A. and Osamura, R. Y. (2003) Vascular networks and endothelial cells in the rat experimental pituitary glands and in the human pituitary adenomas. Microsc. Res. Tech. 60; 231-235.

26. Kaidoh, T., Yasugi, T. and Uehara, Y. (1991) The microvasculature of the 7,12-dimethylbenz (a) anthracene (DMBA)-induced rat mammary tumour. I. Vascular patterns as visualized by scanning electron microscopy of corrosion casts. Virchows Arch. A Pathol. Anat. Histopathol. 418; 111-117.

27. Kakeji, Y. and Teicher, B. A. (1997) Preclinical studies of the combination of angiogenic inhibitors with cytotoxic agents. Invest. New Drugs. 15; 39-48.

28. Kerbel, R. S., Viloria-Petit, A., Klement, G. and Rak, J. (2000) 'Accidental' anti-angiogenic drugs. anti-oncogene directed signal transduction inhibitors and conventional chemotherapeutic agents as examples. Eur. J. Cancer 36; 1248-1257.

29. Klauber, N., Parangi, S., Flynn, E., Hamel, E. and D'Amato, R. J. (1997) Inhibition of angiogenesis and breast cancer in mice by the microtubule inhibitors 2-methoxyestradiol and taxol. Cancer Res. $57 ; 81-86$.

30. Kokufu, I., Taniguchi, H., Kim, Y. H., Fukuda, K., Yamamoto, M., Yano, T., Yamada, K., Kitano, H. and Fukuda, H. (2000) A case of effective chemotherapy using CAF followed by docetaxel for advanced breast cancer. Gan To Kagaku Ryoho 27; $1577-$ 1580. (in Japanese)

31. Kusaka, M., Sudo, K., Matsutani, E., Kozai, Y., Marui, S., Fujita, T., Ingber, D. and Folkman, J. (1994) Cytostatic inhibition of endothelial cell growth by the angiogenesis inhibitor TNP-470 (AGM-1470). Br. J. Cancer 69; 212-216.

32. Moon, C., Verschraegen, C. F., Bevers, M., Freedman, R., Kudelka, A. P. and Kavanagh, J. J. (2000) Use of docetaxel (Taxotere) in patients with paclitaxel (Taxol) hypersensitivity. Anticancer Drugs 11; 565-568.

33. Moulton, K. S., Heller, E., Konerding, M. A., Flynn, E., Palinski, V. and Folkman, J. (1999) Angiogenesis inhibitors endostatin or TNP-470 reduce intimal neovascularization and plaque growth in apolipoprotein E-deficient mice. Circulation 99; 1726-1732.

34. Nakane, P. K. (1975) Recent progress in the peroxidase-labeled antibody method. Ann. NY Acad. Sci. 234; 203-244.

35. Oikawa, T., Hirotani, K., Shimamura, M., Ashino-Fuse, H. and Iwaguchi, T. (1989) Powerful antiangiopgenic activity of herbimcin A. J. Antibiot. 42; 1202-1204.

36. Olson, T. A., Mohanraj, D., Carson, L. F. and Ramakrishnan, S. (1994) Vascular permeability factor gene expression in normal and neoplastic human ovaries. Cancer Res. 54; 276-280.

37. O'Reilly, M. S., Boehm, T., Shing, Y., Fukai, N., Vasios, G., Lane, W. S., Flynn, E., Birkhead, J. R., Olsen, B. R. and Folkman, J. (1997) Endostatin: an endogenous inhibitor of angiogenesis and tumor growth. Cell 88; 277-285.

38. Pagani, O., Sessa, C., Nole, F., Crivellari, D., Lombardi, D., Thurlimann, B., Hess, D., Borner, M., Bauer, J., Martinelli, G., Graffeo, R., Zucchetti, M., D'Incalci, M. and Goldhirsch, A. (2000) Epidoxorubicin and docetaxel as first-line chemotherapy in patients with advanced breast cancer: a multicentric phase I-II study. Ann. Oncol. 11; 985-991.

39. Pepper, M. S., Ferrara, N., Orci, L. and Montesano, R. (1992) Potent synergism between vascular endothelial growth factor and basic fibroblast growth factor in the induction of angiogenesis in vitro. Biochem. Biophys. Res. Commun. 189; 824-831.

40. Plate, K. H., Breier, G., Weich, H. A. and Risau, W. (1992) Vascular endothelial growth factor is a potential tumour angiogenesis factor in human gliomas in vivo. Nature $359 ; 845-848$. 
41. Schimming, R., Mason, K. A., Hunter, N., Weil, M., Kishi, K. and Milas, L. (1999) Lack of correlation between mitotic arrest or apoptosis and antitumor effect of docetaxel. Cancer Chemother Pharmacol. 43; 165-172.

42. Senger, D. R., Galli, S. J., Dvorak, A. M., Perruzzi, C. A., Harvey, V. S. and Dvorak, H. F. (1983) Tumor cells secrete a vascular permeability factor that promotes accumulation of ascites fluid. Science 219; 983-985.

43. Suzuma, T., Sakurai, T., Yoshimura, G., Umemura, T., Tamaki, T. and Naito, Y. (2000) Paclitaxel-induced remission in docetaxel-refractory anthracycline-pretreated metastatic breast cancer. Anticancer Drugs 11; 569-571.

44. Toi, M., Yamamoto, Y., Imazawa, T., Takayanagi, T., Akutsu, K. and Tominaga, T. (1993) Antitumor effect of the angiogenesis inhibitor AGM-1470 and its combination effect with tamoxifen in DMBA induced mammary tumors in rats. Int. J. Oncol. 3; 525528.

45. Twardowski, P. and Gradishar, W. J. (1997) Clinical trials of antiangiogenic agents. Curr. Opin. Oncol. 9; 584-589.

46. Wild, R., Dhanabal, M., Olson, T. A. and Ramakrishnan, S. (2000) Inhibition of angiogenesis and tumour growth by
VEGF121-toxin conjugate: differential effect on proliferating endothelial cells. Br. J. Cancer 83; 1077-1083.

47. Yasumura, K., Ogawa, K., Nakagawa, Y., Yuasa, Y., Hiramatsu, H., Hiramatsu, K. and Osamura, R. Y. (1997) Dynamic MR imaging and tumor angiogenesis in DMBA-induced rat breast cancer; Three dimensional (3D) reconstructed image analysis of tumor microvessels by confocal laser scanning microscopy. Breast Cancer 4; 291-296.

48. Yoshizawa, J., Mizuno, R., Yoshida, T., Hara, A., Ashizuka, S., Kanai, M., Kuwashima, N., Kurobe, M. and Yamazaki, Y. (2000) Inhibitory effect of TNP-470 on hepatic metastasis of mouse neuroblastoma. J. Surg. Res. 93; 82-87.

49. Zeng, S., Chen, Y. Z., Fu, L., Johnson, K. R. and Fan, W. (2000) In vitro evaluation of schedule-dependent interactions between docetaxel and doxorubicin against human breast and ovarian cancer cells. Clin. Cancer Res. 6; 3766-3773.

50. Zhang, Y., Griffith, E. C., Sage, J., Jacks, T. and Liu, J. O. (2000) Cell cycle inhibition by the anti-angiogenic agent TNP-470 is mediated by 533 and p21WAF1/CIP1. Proc. Natl. Acad. Sci. US A 97: 6427-6432. 